\title{
Polarization State Generation and Detection by VCSELs with Integrated Metasurfaces
}

\author{
Dandan Wen ${ }^{1}$ Jiajun Meng ${ }^{1}$, Jasper Cadusch ${ }^{1}$, and Kenneth B. Crozier ${ }^{1,2^{*}}$ \\ 1 Department of Electrical and Electronic Engineering, The University of Melbourne, Victoria 3010, Australia \\ 2 School of Physics, The University of Melbourne, Victoria 3010, Australia \\ *Email: kcrozier@unimelb.edu.au
}

\begin{abstract}
We experimentally demonstrate vertical-cavity surface-emitting lasers (VCSEL) with integrated plasmonic and dielectric metasurfaces. The metasurfaces shape the polarization of the laser emission from the VCSELs and also enable them to serve as polarization-dependent photodetectors.

OCIS codes: $130.0250,160.3918$
\end{abstract}

\section{Introduction}

VCSELs generate laser beams perpendicular to their top surfaces, which facilitates their chip-level integration. Although most VCSELs do not incorporate a method to control output polarization, in some important applications, such as data communication and optical sensing, stable polarization is beneficial or even necessary. This has motivated research on the polarization control of the VCSELs. Approaches investigated thus far include polarization-dependent gain and/or mirrors, asymmetric resonators, and external optical feedback [1]. Most research on the polarization control of VCSELs has focused on the output state, with much less done on using VCSELs as polarization-dependent detectors. Here, we experimentally demonstrate devices which, under the positive bias, generate beams with prescribed polarization states. When negatively biased, the devices work as polarization-dependent photodetectors that distinguish between orthogonal polarization states.

\section{Results}

Device schematics are shown in Fig.1. The VCSEL comprises 34 pairs of distributed Bragg reflectors (DBRs) on the bottom, the gain region, and 24 DBR pairs on the top. Each DBR pair consists of $\mathrm{Al}_{0.9} \mathrm{GaAs}$ and $\mathrm{Al}_{0.12} \mathrm{GaAs}$ layers. The gain region comprises three quantum wells, each comprising GaAs and $\mathrm{Al}_{0.3} \mathrm{GaAs}$ layers. The VCSEL has transverse dimension of $12 \times 12 \mu \mathrm{m}^{2}$ and is $4.2 \mu \mathrm{m}$ tall. We spin coat a polymer (SU8 3010, $10 \mu \mathrm{m}$ thick) onto the VCSEL to protect the gain area from oxidation and to provide a platform for metasurface integration.
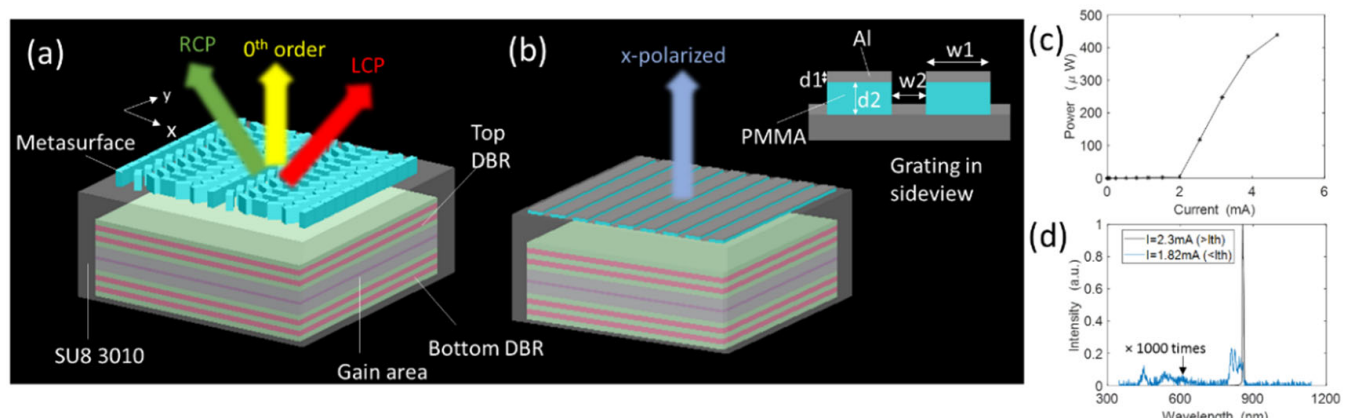

Fig.1. (a) VCSEL with integrated dielectric metasurface (a-Si pillars). Device emits right-handed circularly polarized (RCP) and left-handed circularly polarized (LCP) beams (and some zero-order emission) when operated as a laser. Device can distinguish helicity of light incident upon it when operated as a photodetector. (b) VCSEL with integrated plasmonic metasurface (bi-layer plasmonic grating). Device generates $\mathrm{x}$-polarized beam (when operating as laser) and distinguishes between $\mathrm{x}$ - and $\mathrm{y}$ - polarized incident light (when operating as photodetector). (c) Measured laser power vs drive current. (d) Measured spectra when drive current is below and above threshold. Blue curve has been multiplied by $1000 \times$

As shown in Fig. 1(a), for the VCSEL with the integrated dielectric metasurface, the latter comprises a collection of amorphous silicon (a-Si) nanopillars with rectangular cross sections (width $120 \mathrm{~nm}$, length $270 \mathrm{~nm}$, height 600 $\mathrm{nm})$. The a-Si nanopillars are arranged in a square lattice $(300 \mathrm{~nm}$ period). The orientation of the nanopillars varies by $30^{\circ}$ between unit cells in the x-direction. Such a design enables the metasurface to work as a polarization beam splitter $[2,3]$, decomposing the output light from the VCSEL into left-handed and right-handed circularly polarized (LCP and RCP) beams that propagate in different directions $\left( \pm 28.2^{\circ}\right)$. Our device also acts as a polarization-dependent photodetector. I.e. when illuminated with a beam at a tilted angle, the transmittance of the metasurface (and thus the photocurrent measured from the negatively-biased VCSEL) will be different for LCP and RCP. In Fig. 1b, a schematic diagram of our VCSEL with integrated plasmonic metasurface is shown. The 
latter comprises a double-layer grating fabricated on the VCSEL. Here we have $w 1=150 \mathrm{~nm}, \mathrm{w} 2=80 \mathrm{~nm}, \mathrm{~d} 1=60 \mathrm{~nm}$, $\mathrm{d} 2=200 \mathrm{~nm}$ (see inset Fig. 1b). The grating functions as a polarizer and light from the VCSEL is thus X-polarized. This device also functions as a polarization-dependent photodetector (when negatively biased). The measured power vs current and spectra of the VCSEL before integration of the metasurface are shown as Fig. 1c-d.

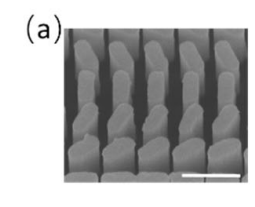

(e)

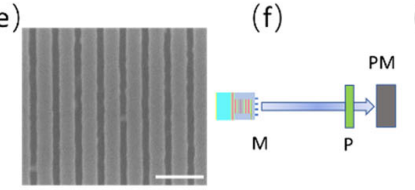

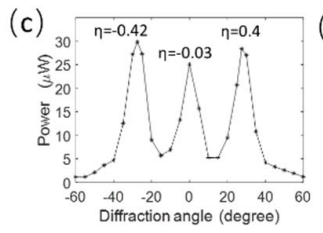

(g)

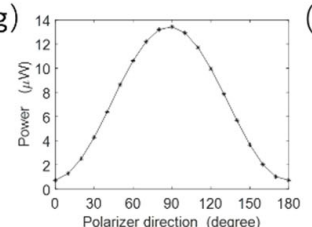

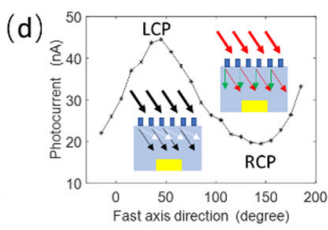

(h)

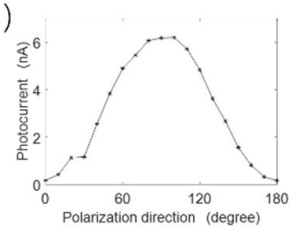

Fig.2. (a) SEM of dielectric metasurface (a-Si pillars) on VCSEL. Scalebar: $500 \mathrm{~nm}$. (b) Experimental setup to verify polarization state of output light. M: metasurface. QW: quarter-wave plate. P: polarizer. PM: power meter. (c) Measured power versus diffraction angle. Degree of circular polarization (DOP, $\eta$ ) of the $0^{\text {th }}$ and $\pm 1^{\text {st }}$ orders are measured and labelled in plot. (d) Experimental results when device operated as detector (biased at -2V). Photocurrent vs QW angle shows peak/dip when illumination is LCP/RCP. (e) SEM of plasmonic metasurface on VCSEL. Scalebar: $500 \mathrm{~nm}$. (f) Experimental setup for polarization measurement. (g) Experimental results when device operated as laser. Measured power vs P direction. (h) Experimental results when device operated as detector. Measured photocurrent vs polarization of input light (linearly-polarized, at $850 \mathrm{~nm}$ ).

VCSEL devices with integrated dielectric and plasmonic metasurfaces are fabricated as follows. We first make the contact rings on the top DBR, then dry etch to form the mesa. We next spin coat SU8 to planarize the device and make a P-electrode (connected to the ring) on it. We also make an N-electrode on the backside of the substrate. After that, we spin on more SU8 and make a metasurface on it. For the dielectric metasurface, the latter is achieved via a-Si deposition, e-beam lithography and dry etching. For the plasmonic metasurface, the metasurface is made by e-beam lithography and aluminum deposition. A scanning electron micrograph (SEM) of the former is shown as Fig. 2a. The experimental setup for characterizing the polarization of light from the device is shown as Fig. $2 \mathrm{~b}$. The measured optical power versus diffraction angle (Fig. 2c) shows that most of the light from the VCSEL is diffracted to the \pm 1 orders. Here we have $\eta=\left(I_{R C P}-I_{L C P}\right) /\left(I_{R C P}+I_{L C P}\right)$ as the degree of circular polarization (DOP), where $\mathrm{I}_{\mathrm{RCP}}\left(\mathrm{I}_{\mathrm{LCP}}\right)$ denotes the power of the RCP(LCP) components. The values we measure for $\eta$ (Fig. $2 \mathrm{c}$ ) show that the +1 and -1 orders are different in helicity (i.e. $\eta$ takes opposite sign). We next apply negative bias to the device and illuminate it with light from a laser diode $(850 \mathrm{~nm})$ that has passed through a polarizer and quarter waveplate. This illumination is at an angle that corresponds to the +1 order. It can be seen that the photocurrent is strongly dependent on the input polarization state (Fig. 2d). This can be understood as follows. When the incident light is LCP (bold black arrow, inset Fig. 2d), the portion of the beam transmitted by the metasurface that is not converted (slim black arrow) propagates along the original direction, while the portion converted (to RCP, white arrow) changes direction (due to metasurface phase gradient), illuminates the sidewall of the VCSEL mesa and is coupled into the gain area. When the incident light is RCP (bold red arrow), the converted part (green arrow) is normally-incident on the VCSEL and largely is reflected back by the top DBRs. The photocurrent is thus strongly dependent on the polarization state of the incident light. An SEM image of the plasmonic metasurface that is integrated onto a VCSEL is shown as Fig. 2e. We use an experimental set-up (Fig. 2f) to characterize the polarization of light from the device and find that it is almost completely linearly polarized (Fig $2 \mathrm{~g}$ ). We next apply negative bias to our device and operate it as a photodetector. We illuminate it at normal incidence with linearly polarized light from a laser diode (at $850 \mathrm{~nm}$ ) and measure the photocurrent as a function of polarization direction (varied using a half-wave plate). The results (Fig. 2h) demonstrate that the photocurrent is strongly dependent on the polarization of the incident light.

In conclusion, we demonstrate electrically-pumped VCSEL devices that represent fully-contained systems for polarization state generation and detection. In lasing mode, the dielectric metasurface device generates two beams with orthogonal polarizations while the plasmonic metasurface device generates linearly polarized light. In detector mode, the dielectric metasurface device distinguishes the handedness of the incoming light, while the plasmonic metasurface device has a large extinction ratio for orthogonal linear polarizations.

\section{References}

[1]. R. Michalzik, VCSELs: fundamentals, technology and applications of vertical-cavity surface-emitting lasers (Springer, 2012).

[2]. M. Khorasaninejad, and K. B. Crozier, "Silicon nanofin grating as a miniature chirality-distinguishing beam-splitter," Nat. Commun. 5, 5386 (2014).

[3]. D. Wen, F. Yue, S. Kumar, Y. Ma, M. Chen, X. Ren, P. E. Kremer, B. D. Gerardot, M. R. Taghizadeh, G. S. Buller, and X. Chen, "Metasurface for characterization of the polarization state of light," Opt. Express 23, 10272-10281 (2015). 


\section{University Library}

\section{- M M I N E R VA A gateway to Melbourne's research publications}

Minerva Access is the Institutional Repository of The University of Melbourne

Author/s:

Wen, D;Meng, J;Cadusch, J;Crozier, KB

Title:

Polarization State Generation and Detection by VCSELs with Integrated Metasurfaces

Date:

2020-01-01

Citation:

Wen, D., Meng, J., Cadusch, J. \& Crozier, K. B. (2020). Polarization State Generation and Detection by VCSELs with Integrated Metasurfaces. 2020 CONFERENCE ON LASERS AND ELECTRO-OPTICS (CLEO), Part F181-CLEO-AT 2020, OSA \& IEEE. https://doi.org/10.1364/ cleo_at.2020.am4k.5.

Persistent Link:

http://hdl.handle.net/11343/294869 